Tohoku Math. J.

56 (2004), 271-287

\title{
AN ELEMENTARY PROOF OF GLOBAL OR ALMOST GLOBAL EXISTENCE FOR QUASI-LINEAR WAVE EQUATIONS
}

\author{
KUNIO HidANO
}

(Received October 21, 2002, revised May 27, 2003)

\begin{abstract}
We give a new, elementary proof of the global or almost global existence theorem of S. Klainerman. Our result also covers the almost global existence theorem of M. Keel, F. Smith, and C. D. Sogge. The proof is carried out in line with S. Klainerman and T. C. Sideris.
\end{abstract}

1. Introduction. Since the classic work of John [9] on the finite-time blow-up of smooth solutions to quadratic quasi-linear wave equations in three space dimensions with small data, a large amount of effort has been made to clarify the relation among the life-span (the maximal existence time of unique local solutions), the structure of quadratic nonlinear terms, and the number of space dimensions. One of the most striking results in this direction is due to Klainerman [15], [17], who proved the global existence theorem in space dimensions $n \geq 4$ and the "almost global" existence theorem in three space dimensions, for small data. As is explained widely in excellent monographs such as [3], [7], [11], [22], [26] and [28], the heart of the method of Klainerman is an effective use of the Killing vector fields as well as the scaling operator $t \partial_{t}+x \cdot \nabla$ to prove global Sobolev inequalities in the Minkowski space $\boldsymbol{R}^{n+1}$, known by the name of the Klainerman inequality [17]. Thanks to the good commutation relation between the d'Alembertian $\partial_{t}^{2}-\Delta$ and the generators of the Poincaré group, the Klainerman inequality plays a central role in the proof of global or almost global existence of small amplitude solutions.

Interestingly enough, the method of vector fields has been evolving into new versions, as is seen in, e.g., [2], [8], [10], [14], [18], [19], [23]-[25], [27] and [29] from motives for studying the Cauchy problem of nonlinear elastic equations as well as the initial-boundary value problem of quasi-linear wave equations in an exterior of a compact, nontrapping or star-shaped set with a smooth boundary. See also technical innovations in Keel, Smith and Sogge [13] and Metcalfe [20], where they have further evolved the method of vector fields to consider semi-linear wave equations in exterior domains as well as in the Minkowski space. The common feature of the revised methods lies in that the use of Lorentz boosts, which is ill-suited for the analysis of these problems, is completely avoided. The lack of Lorentz

2000 Mathematics Subject Classification. 35L70.

Key words and phrases. Quasi-linear wave equation, initial value problem.

The author is grateful to Kazuyoshi Yokoyama for pointing out a mistake in the first manuscript. Thanks are also due to the referee for helpful comments. He is supported by the Grant-in-Aid for Scientific Research (No. 13740101), the Ministry of Education, Culture, Sports, Science and Technology. 
boosts in the list of vector fields are compensated for by direct estimates of the fundamental solution [2], [8], [10], [14], [19], [29] or by somewhat weak Sobolev-type inequalities [6], [18], [23]-[25] to get some $L^{\infty}$-decay estimates of local solutions. The main purpose of this paper is to develop the recent superb version of Klainerman and Sideris [18], and cover the global or almost global existence theorem of Klainerman without the use of the Lorentz boosts. Since the operator $\Gamma^{a}$ contains at most one scaling operator in the definition of the generalized energy for $n=3$ in (2.4), our almost global existence theorem actually covers Theorem 1.2 of Keel, Smith and Sogge [14]. The author believes that the present method will evolve into an efficient technique of the analysis of the initial-boundary value problem for quasi-linear wave equations in an exterior of a 3-D compact and star-shaped set with a smooth boundary.

We describe technical features in the present paper at length. Our analysis is in line with that of Sideris [24] and Sideris and Tu [25], who have significantly improved an intriguing and striking method of Klainerman and Sideris [18]. Via the Klainerman-Sideris inequality (see (5.1) below) we can obtain some weighted $L^{2}\left(\boldsymbol{R}^{n}\right)$-estimates of the second and higher-order derivatives, as long as a local solution is sufficiently small in a suitable sense. The weight involved has the form of $\langle c t-r\rangle$ for a wave-propagation speed $c$, and the time decay thereby obtained of the $L^{2}$-norms compensates efficiently for some weak time decay of the $L^{\infty}$-norms which can be obtained via the Sobolev-type inequality such as (4.2) below. It is noted that we can handle semi-linear terms $H^{k}(u, u)$ of (3.3) by our analysis with an effective application of the Hardy inequality as in Hidano [6], though the Klainerman-Sideris inequality does not lead to weighted $L^{2}\left(\boldsymbol{R}^{n}\right)$-estimates of the first derivatives. We also add two comments. First, it is possible to deal with non-divergence nonlinear terms by our analysis thanks to significant technical improvements of Sideris [24], though the innovative paper of Klainerman and Sideris [18] discussed only nonlinear terms of the divergence form. Secondly, the present analysis needs no direct estimates of the fundamental solution and, employing our techniques, we have recently given a unified proof of the previous excellent results in two or three space dimensions of Agemi and Yokoyama [2], Hoshiga and Kubo [8], Kovalyov [19] and Yokoyama [29] whose proofs built heavily upon direct estimates of the fundamental solution. See [6].

This paper is organized as follows. In the next section we give the notation used in this paper. In Section 3 the main results are stated. Section 4 is devoted to the proof of some Sobolev-type inequalities. After weighted $L^{2}$-norms are shown to be bounded by generalized energies in Section 5, we complete the energy integral argument in Sections 6 and 7 to prove almost global and global existence theorem.

2. Notation. We explain the notation used in this paper. Let $n$ denote the space dimensions. We shall consider systems of $m$ quasi-linear equations. Repeated indices are summed if lowered and raised. Greek indices range from 0 to $n$, and Latin indices from 1 to $m$. Points in $\boldsymbol{R}_{+}^{n+1}$ are denoted by $\left(x^{0}, x^{1}, \ldots, x^{n}\right)=(t, x)$. In addition to the usual partial differential operators $\partial_{\alpha}=\partial / \partial x^{\alpha}(\alpha=0, \ldots, n)$ with the abbreviation $\partial=\left(\partial_{0}, \partial_{1}, \ldots, \partial_{n}\right)=$ 
$\left(\partial_{t}, \nabla\right)$, we shall use the generators of the Euclid rotations $\Omega=\left(\Omega_{12}, \ldots, \Omega_{1 n}, \Omega_{23}, \ldots\right.$, $\left.\Omega_{n-1 n}\right)$ with $\Omega_{j k}=x^{j} \partial_{k}-x^{k} \partial_{j}(1 \leq j<k \leq n)$, and the generators of the spacetime scaling $S=x^{\alpha} \partial_{\alpha}$. The set of these $v=\left(n^{2}+n+4\right) / 2$ vector fields is denoted by $\Gamma=\left(\Gamma_{0}, \Gamma_{1}, \ldots, \Gamma_{\nu-1}\right)=(\partial, \Omega, S)$. We also denote $\Gamma \backslash\{S\}$ by $\bar{\Gamma}=\left(\Gamma_{0}, \Gamma_{1}, \ldots, \Gamma_{\nu-2}\right)=$ $(\partial, \Omega)$. For multi-indices $a=\left(a_{0}, \ldots, a_{v-1}\right)$ and $b=\left(b_{0}, \ldots, b_{v-2}\right)$, we denote

$$
\Gamma^{a}=\Gamma_{0}^{a_{0}} \cdots \Gamma_{\nu-1}^{a_{v-1}}, \quad \bar{\Gamma}^{b}=\Gamma_{0}^{b_{0}} \cdots \Gamma_{\nu-2}^{b_{v-2}} .
$$

The D'Alembertian, which acts on vector-valued functions $u: \boldsymbol{R}_{+}^{n+1} \rightarrow \boldsymbol{R}^{m}$, is denoted by

$$
\square=\operatorname{diag}\left(\square_{1}, \ldots, \square_{m}\right), \quad \square_{k}=\frac{\partial^{2}}{\partial t^{2}}-c_{k}^{2} \Delta .
$$

Associated with this operator, the energy is defined as

$$
E_{1}(u(t))=\frac{1}{2} \sum_{k=1}^{m} \int_{\boldsymbol{R}^{n}}\left|\partial_{t} u^{k}(t, x)\right|^{2}+c_{k}^{2}\left|\nabla u^{k}(t, x)\right|^{2} d x .
$$

We also introduce two types of generalized energy as

$$
\begin{gathered}
E_{\kappa}(u(t))=\sum_{\substack{|a| \leq \kappa-1 \\
\mid a_{\nu}-1 \leq 1}} E_{1}\left(\Gamma^{a} u(t)\right) \quad \text { if } n=3, \\
E_{\kappa}(u(t))=\sum_{|a| \leq \kappa-1} E_{1}\left(\Gamma^{a} u(t)\right) \quad \text { if } n \geq 4, \\
\bar{E}_{\kappa}(u(t))=\sum_{|a| \leq \kappa-1} E_{1}\left(\bar{\Gamma}^{a} u(t)\right)
\end{gathered}
$$

for $\kappa=2,3, \ldots$.

Two types of auxiliary norm

$$
\begin{gathered}
M_{\kappa}(u(t))=\left\{\begin{array}{l}
\sum_{k=1}^{m} \sum_{|a|=2} \sum_{|b| \leq \kappa-2}\left\|\left\langle c_{k} t-r\right\rangle \partial^{a} \bar{\Gamma}^{b} u^{k}(t)\right\|_{L^{2}\left(\boldsymbol{R}^{3}\right)}, n=3, \\
\sum_{k=1}^{m} \sum_{|a|=2} \sum_{|b| \leq \kappa-2}\left\|\left\langle c_{k} t-r\right\rangle \partial^{a} \Gamma^{b} u^{k}(t)\right\|_{L^{2}\left(\boldsymbol{R}^{n}\right)}, n \geq 4,
\end{array}\right. \\
\bar{M}_{\kappa}(u(t))=\sum_{k=1}^{m} \sum_{|a|=2} \sum_{|b| \leq \kappa-2}\left\|\left\langle c_{k} t-r\right\rangle \partial^{a} \bar{\Gamma}^{b} u^{k}(t)\right\|_{L^{2}\left(\boldsymbol{R}^{n}\right)}
\end{gathered}
$$

(hence $\bar{M}_{\kappa}(u(t))=M_{\kappa}(u(t))$ for $n=3$ ) will play an intermediary role in the energy integral argument below. Here and later on as well we use the notation $\langle A\rangle=\sqrt{1+|A|^{2}}$ for a scalar or vector $A$. For simplicity we shall often denote the $L^{p}\left(\boldsymbol{R}^{n}\right)$-norm by $\|\cdot\|_{L^{p}}$.

3. Results. We consider the Cauchy problem for a system of quasi-linear wave equations

$$
\square u=F\left(\partial u, \partial^{2} u\right) \quad \text { in } \quad \boldsymbol{R}_{+}^{1+n}
$$


( $n \geq 3$ ) subject to the smooth, compactly supported initial data

$$
u(0)=\varphi, \quad \partial_{t} u(0)=\psi .
$$

We may suppose that the nonlinear term $F$ is quadratic, because higher-order terms have no influence over the theory of large-time existence of small amplitude solutions. We assume the $k$-th component of the vector function $F$ to be of the form $F^{k}\left(\partial u, \partial^{2} u\right)=G^{k}(u, u)+$ $H^{k}(u, u)$, where

$$
G^{k}(u, v)=G_{i j}^{k, \alpha \beta \gamma} \partial_{\alpha} u^{i} \partial_{\beta} \partial_{\gamma} v^{j}, \quad H^{k}(u, v)=H_{i j}^{k, \alpha \beta} \partial_{\alpha} u^{i} \partial_{\beta} v^{j}
$$

for real constants $G_{i j}^{k, \alpha \beta \gamma}, H_{i j}^{k, \alpha \beta}$. Since our proof is based on the energy integral method, we naturally assume the symmetry condition

$$
G_{i j}^{k, \alpha \beta \gamma}=G_{i j}^{k, \alpha \gamma \beta}=G_{i k}^{j, \alpha \beta \gamma} .
$$

The main theorems of this paper are stated as follows.

THEOREM 3.1. Let $n=3$ and $\kappa \geq 6$. There exists a constant $\delta>0$ with the following property: If the initial data satisfy $E_{\kappa}^{1 / 2}(u(0))<\varepsilon$ for $\varepsilon<\delta$, then the existence time of a unique, local smooth solution to (3.1) and (3.2) exceeds $A \exp [B / \varepsilon]$, where $A$ and $B$ are positive constants independent of $\varepsilon$.

THEOREM 3.2. Let $n \geq 4$ and let $\kappa$ be large so that

$$
\left[\frac{\kappa}{2}\right]+\left[\frac{n}{2}\right]+2 \leq \kappa-\left[\frac{n}{2}\right]-1 .
$$

There exist positive constants $\delta$ and $C_{0}$ with the following property: If the initial data satisfy

$$
E_{\mu}^{1 / 2}(u(0)) \exp \left[C_{0} E_{\kappa}^{1 / 2}(u(0))\right]<\delta\left(\mu \equiv \kappa-\left[\frac{n}{2}\right]-1\right),
$$

then there exists a unique, smooth global solution to (3.1) and (3.2).

4. Preliminaries. In addition to the well-known facts

$$
[\partial, \square]=0, \quad[\Omega, \square]=0, \quad[S, \square]=-2 \square,
$$

we shall need the following Sobolev-type inequalities.

LEMMA 4.1. (i) Let $n \geq 3$. The inequality

$$
\langle r\rangle^{(n / 2)-1}\left\langle c_{j} t-r\right\rangle\left|\partial u^{j}(t, x)\right| \leq C \bar{E}_{[n / 2]+1}^{1 / 2}(u(t))+C \bar{M}_{[n / 2]+2}(u(t))
$$

holds.

(ii) Let $n=3$. The inequality

$$
\langle r\rangle|\partial u(t, x)| \leq C \bar{E}_{3}^{1 / 2}(u(t))
$$

holds. 
(iii) Let $n=4$ and $0 \leq \varepsilon<1 / 2$. The inequality

$$
\langle r\rangle^{1+\varepsilon}|u(t, x)| \leq C \sum_{|a| \leq 2}\left\|\Omega^{a} u(t)\right\|_{L^{2}}^{\varepsilon}\left\|\Omega^{a} u(t)\right\|_{\dot{H}_{2}^{1}}^{1-\varepsilon}+C \sum_{|a|=1}^{3}\left\|\partial_{x}^{a} u(t)\right\|_{L^{2}}
$$

holds.

(iv) Let $n \geq 3$. The inequality

$$
\langle r\rangle^{(n / 2)-1}|u(t, x)| \leq C \sum_{|a| \leq[n / 2]}\left\|\Omega^{a} u(t)\right\|_{\dot{H}_{2}^{1}}+C \sum_{|a|=1}^{[n / 2]+1}\left\|\partial_{x}^{a} u(t)\right\|_{L^{2}}
$$

holds.

PROOF. The inequality (4.3) was shown by Sideris (see (3.20b) of [24], and also (6.2) of Sideris and $\mathrm{Tu}[25]$ ). The proof of (4.4) starts with the following radius-angular mixed-norm inequality which has been shown in Hidano [4]:

$$
r^{(n / 2)-s}\left(\int_{S^{n-1}}|v(r \omega)|^{2} d \omega\right)^{1 / 2} \leq C\|v\|_{\dot{H}_{2}^{s}}, \quad \frac{1}{2}<s<\frac{n}{2} .
$$

Set $s=1-\varepsilon$. It then follows from the Sobolev embedding on the unit sphere $S^{3}$ in $\boldsymbol{R}^{4}$ that

$$
\begin{aligned}
r^{1+\varepsilon}|u(t, x)| & \leq C \sum_{|a| \leq 2} r^{1+\varepsilon}\left(\int_{S^{3}}\left|\Omega^{a} u(t, x)\right|^{2} d \omega\right)^{1 / 2} \\
& \leq C \sum_{|a| \leq 2}\left\|\Omega^{a} u(t)\right\|_{\dot{H}_{2}^{1-\varepsilon}} \leq C \sum_{|a| \leq 2}\left\|\Omega^{a} u(t)\right\|_{L^{2}}^{\varepsilon}\left\|\Omega^{a} u(t)\right\|_{\dot{H}_{2}^{1}}^{1-\varepsilon}, r>0 .
\end{aligned}
$$

Let us introduce a smooth cut-off function $\Phi \in C_{0}^{\infty}\left(\boldsymbol{R}^{4}\right), \Phi=1$ for $|x| \leq 1, \Phi=0$ for $|x| \geq 2$. For $|x| \leq 1$ we see

$$
\begin{aligned}
|u(t, x)| & =|\Phi(x) u(t, x)| \leq C\|\Phi u(t)\|_{H^{3}} \\
& \leq C\|u(t)\|_{L^{2}(1<|x|<2)}+C \sum_{|a|=1}^{3}\left\|\partial_{x}^{a} u(t)\right\|_{L^{2}} \\
& \leq C \sup _{1<|x|<2}|u(t, x)|+C \sum_{|a|=1}^{3}\left\|\partial_{x}^{a} u(t)\right\|_{L^{2}} \\
& \leq C \sum_{|a| \leq 2}\left\|\Omega^{a} u(t)\right\|_{L^{2}}^{\varepsilon}\left\|\Omega^{a} u(t)\right\|_{\dot{H}_{2}^{1}}^{1-\varepsilon}+C \sum_{|a|=1}^{3}\left\|\partial_{x}^{a} u(t)\right\|_{L^{2}},
\end{aligned}
$$

which together with (4.7) leads to (4.4).

In order to show (4.5) we make use of the following inequality due to Nakanishi (see Proposition 3.7 of [21]):

$$
r^{(n / p)-1}\left(\int_{S^{n-1}}|v(r \omega)|^{q} d \omega\right)^{1 / q} \leq C\|v\|_{\dot{H}_{p}^{1}},
$$


for $1 \leq p<n, n / p-(n-1) / q=1$. Choosing $p=2$ and modifying (4.7) and (4.8) properly, we can easily show (4.5).

The inequality (4.2) remains to be proved. Getting back to (4.9) with $p=2$, we see for $r>0$

$$
\begin{aligned}
r^{(n / 2)-1}\left|\left\langle c_{j} t-r\right\rangle \partial u^{j}(t, x)\right| & C r^{(n / 2)-1} \sum_{|a| \leq[n / 2]}\left(\int_{S^{n-1}}\left|\Omega^{a}\left(\left\langle c_{j} t-r\right\rangle \partial u^{j}(t, x)\right)\right|^{q} d \omega\right)^{1 / q} \\
\leq & C \sum_{|a| \leq[n / 2]}\left\|\left\langle c_{j} t-r\right\rangle \Omega^{a} \partial u^{j}(t)\right\|_{\dot{H}_{2}^{1}} \\
\leq & C \sum_{|a| \geq 1} \sum_{|a|+|b| \leq[n / 2]+1}\left\|\left\langle c_{j} t-r\right\rangle \partial^{a} \Omega^{b} u^{j}(t)\right\|_{\dot{H}_{2}^{1}} \\
\leq & C \sum_{|a| \geq 1|a|+|b| \leq[n / 2]+1}\left\|\partial^{a} \Omega^{b} u^{j}(t)\right\|_{L^{2}} \\
& +C \sum_{|a| \geq 2} \sum_{|a|+|b| \leq[n / 2]+2}\left\|\left\langle c_{j} t-r\right\rangle \partial^{a} \Omega^{b} u^{j}(t)\right\|_{L^{2}} .
\end{aligned}
$$

Let us again introduce a smooth cut-off function $\Phi \in C_{0}^{\infty}\left(\boldsymbol{R}^{n}\right), \Phi=1$ for $|x| \leq 1, \Phi=0$ for $|x| \geq 2$. For $r \leq 1$ we see, assuming $c_{j} t \geq 3$ without loss of generality,

$$
\begin{aligned}
\mid\left\langle c_{j} t\right. & -r\rangle \partial u^{j}(t, x)\left|\leq\left(1+c_{j} t\right)\right| \Phi(x) \partial u^{j}(t, x) \mid \\
\leq & C\left(1+c_{j} t\right)\left\|\Phi \partial u^{j}(t)\right\|_{H^{[n / 2]+1}} \leq C\left(1+c_{j} t\right) \sum_{|a|=1}^{[n / 2]+1}\left\|\partial_{x}^{a}\left(\Phi \partial u^{j}(t)\right)\right\|_{L^{2}} \\
\leq & C\left(1+c_{j} t\right)\left(\sum_{|a|=1}^{[n / 2]+1}\left\|\partial_{x}^{a} \partial u^{j}(t)\right\|_{L^{2}(|x|<2)}+\sup _{1<|x|<2}\left|\partial u^{j}(t, x)\right|\right) \\
\leq & C \sum_{|a|=2}^{[n / 2]+2}\left\|\left\langle c_{j} t-r\right\rangle \partial^{a} u^{j}(t)\right\|_{L^{2}}+C \sum_{|a| \geq 1|a|+|b| \leq[n / 2]+1}\left\|\partial^{a} \Omega^{b} u^{j}(t)\right\|_{L^{2}} \\
& +C \sum_{|a| \geq 2|a|+|b| \leq[n / 2]+2}\left\|\left\langle c_{j} t-r\right\rangle \partial^{a} \Omega^{b} u^{j}(t)\right\|_{L^{2}},
\end{aligned}
$$

where we have employed (4.10) at the last inequality. The inequality (4.2) is an immediate consequence of (4.10) and (4.11).

The next corollary follows directly from (4.4) and (4.5).

COROLlary 4.2. (i) Let $n=4$ and $0 \leq \varepsilon<1 / 2$. The inequality

$$
\langle r\rangle^{1+\varepsilon}|\partial u(t, x)| \leq C \bar{E}_{4}^{1 / 2}(u(t))
$$

holds. 
(ii) Suppose $n \geq 5$. The inequality

$$
\langle r\rangle^{(n / 2)-1}|\partial u(t, x)| \leq C \bar{E}_{[n / 2]+2}^{1 / 2}(u(t))
$$

holds.

5. Weighted $L^{2}$-estimates. Since weighted $L^{2}$-norms $\bar{M}_{\kappa}(u(t))$ appear on the righthand side of the Sobolev-type inequalities presented in the previous section, it is necessary to bound the weighted norms $M_{\kappa}(u(t))$ or $\bar{M}_{\kappa}(u(t))$ by $E_{\kappa}^{1 / 2}(u(t))$ for the completion of the energy integral argument. The next crucial inequality, which is due to Klainerman and Sideris, is the starting point of our proof.

LEMMA 5.1 (Klainerman-Sideris inequality). Let $\kappa \geq 2$.

(i) Let $n=3$. The inequality

$$
M_{\kappa}(u(t)) \leq C E_{\kappa}^{1 / 2}(u(t))+C \sum_{|a| \leq \kappa-2}\left\|(t+r) \square \bar{\Gamma}^{a} u(t)\right\|_{L^{2}}
$$

holds for any smooth function $u: \boldsymbol{R}_{+}^{3+1} \rightarrow \boldsymbol{R}^{m}$ with the finite right-hand side.

(ii) Let $n \geq 4$. The inequality

$$
M_{\kappa}(u(t)) \leq C E_{\kappa}^{1 / 2}(u(t))+C \sum_{|a| \leq \kappa-2}\left\|(t+r) \square \Gamma^{a} u(t)\right\|_{L^{2}}
$$

holds for any smooth function $u: \boldsymbol{R}_{+}^{n+1} \rightarrow \boldsymbol{R}^{m}$ with the finite right-hand side.

Proof. See Lemma 3.1 of Klainerman and Sideris [18] and Lemma 7.1 of Sideris and $\mathrm{Tu}$ [25]. Note that their proof is obviously valid for all $n \geq 2$.

LEMMA 5.2. Let $u$ be a smooth solution of (3.1) and (3.2). Set $\kappa^{\prime}=[(\kappa-1) / 2]+$ $[n / 2]+2$. Then for all $|a| \leq \kappa-2$

$$
\begin{aligned}
\|(t+ & r) \square \hat{\Gamma}^{a} u(t) \|_{L^{2}} \\
& \leq C E_{\kappa^{\prime}}^{1 / 2}(u(t)) E_{\kappa}^{1 / 2}(u(t))+C M_{\kappa^{\prime}}(u(t)) E_{\kappa}^{1 / 2}(u(t))+C E_{\kappa^{\prime}}^{1 / 2}(u(t)) M_{\kappa}(u(t)) .
\end{aligned}
$$

Here and later on as well $\hat{\Gamma}^{a}=\bar{\Gamma}^{a}$ for $n=3, \hat{\Gamma}^{a}=\Gamma^{a}$ for $n \geq 4$.

PROOF. We may focus on the estimate of the $L^{2}$-norm of $t \square \hat{\Gamma}^{a} u$, because that of $r \square \hat{\Gamma}^{a} u$ is treated in an easier way. Set $p=[(\kappa-1) / 2]$. It is necessary to estimate the contribution from the quasi-linear parts

$$
t\left\|\partial \hat{\Gamma}^{b} u^{i}(t) \partial^{2} \hat{\Gamma}^{c} u^{j}(t)\right\|_{L^{2}}, \quad|b|+|c| \leq \kappa-2
$$

as well as the contribution from the semi-linear parts

$$
t\left\|\partial \hat{\Gamma}^{b} u^{i}(t) \partial \hat{\Gamma}^{c} u^{j}(t)\right\|_{L^{2}}, \quad|b|+|c| \leq \kappa-2 .
$$


Set $c_{0}=\min \left\{c_{j} / 2 ; j=1, \ldots, m\right\}$. We shall start with the estimate of (5.5). Let us assume $|b| \leq p$ without loss of generality. It follows from (4.2), (4.3) and (4.5) that

$$
\begin{aligned}
& t\left\|\partial \hat{\Gamma}^{b} u^{i} \partial \hat{\Gamma}^{c} u^{j}\right\|_{L^{2}} \\
& \leq C\left\|\left\langle c_{i} t-r\right\rangle \partial \hat{\Gamma}^{b} u^{i} \partial \hat{\Gamma}^{c} u^{j}\right\|_{L^{2}\left(r<c_{0} t\right)}+C\left\|r \partial \hat{\Gamma}^{b} u^{i} \partial \hat{\Gamma}^{c} u^{j}\right\|_{L^{2}\left(r>c_{0} t\right)} \\
& \leq C\left(\left\|\left\langle c_{i} t-r\right\rangle \partial \hat{\Gamma}^{b} u^{i}\right\|_{L^{\infty}}+\left\|r \partial \hat{\Gamma}^{b} u^{i}\right\|_{L^{\infty}}\right)\left\|\partial \hat{\Gamma}^{c} u^{j}\right\|_{L^{2}} \\
& \leq C\left(E_{|b|+[n / 2]+2}^{1 / 2}(u(t))+M_{|b|+[n / 2]+2}(u(t))\right) E_{|c|+1}^{1 / 2}(u(t)) \\
& \leq C\left(E_{p+[n / 2]+2}^{1 / 2}(u(t))+M_{p+[n / 2]+2}(u(t))\right) E_{\kappa-1}^{1 / 2}(u(t)) \\
& \leq C\left(E_{\kappa^{\prime}}^{1 / 2}(u(t))+M_{\kappa^{\prime}}(u(t))\right) E_{\kappa-1}^{1 / 2}(u(t)) \text {. }
\end{aligned}
$$

For the estimate of (5.4) we separate two cases: $|b| \leq p$ or $|c| \leq p-1$. For the former case the estimate is carried out as

$$
\begin{aligned}
t\left\|\partial \hat{\Gamma}^{b} u^{i} \partial^{2} \hat{\Gamma}^{c} u^{j}\right\|_{L^{2}} & \leq C\left\|\partial \hat{\Gamma}^{b} u^{i}\left\langle c_{j} t-r\right\rangle \partial^{2} \hat{\Gamma}^{c} u^{j}\right\|_{L^{2}\left(r<c_{0} t\right)}+C\left\|r \partial \hat{\Gamma}^{b} u^{i} \partial^{2} \hat{\Gamma}^{c} u^{j}\right\|_{L^{2}\left(r>c_{0} t\right)} \\
& \leq C\left\|\partial \hat{\Gamma}^{b} u^{i}\right\|_{L^{\infty}}\left\|\left\langle c_{j} t-r\right\rangle \partial^{2} \hat{\Gamma}^{c} u^{j}\right\|_{L^{2}}+C\left\|r \partial \hat{\Gamma}^{b} u^{i}\right\|_{L^{\infty}}\left\|\partial^{2} \hat{\Gamma}^{c} u^{j}\right\|_{L^{2}} \\
& \leq C E_{|b|+[n / 2]+2}^{1 / 2}(u(t))\left(M_{|c|+2}(u(t))+E_{|c|+2}^{1 / 2}(u(t))\right) \\
& \leq C E_{\kappa^{\prime}}^{1 / 2}(u(t))\left(M_{\kappa}(u(t))+E_{\kappa}^{1 / 2}(u(t))\right) .
\end{aligned}
$$

On the other hand, for $|c| \leq p-1$, we proceed as

$$
\begin{aligned}
t \| \partial \hat{\Gamma}^{b} & u^{i} \partial^{2} \hat{\Gamma}^{c} u^{j} \|_{L^{2}} \\
& \leq C\left\|\partial \hat{\Gamma}^{b} u^{i}\right\|_{L^{2}}\left(\left\|\left\langle c_{j} t-r\right\rangle \partial^{2} \hat{\Gamma}^{c} u^{j}\right\|_{L^{\infty}}+\left\|r \partial^{2} \hat{\Gamma}^{c} u^{j}\right\|_{L^{\infty}}\right) \\
& \leq C E_{|b|+1}^{1 / 2}(u(t))\left(E_{|c|+[n / 2]+3}^{1 / 2}(u(t))+M_{|c|+[n / 2]+3}(u(t))\right) \\
& \leq C E_{\kappa}^{1 / 2}(u(t))\left(E_{\kappa^{\prime}}^{1 / 2}(u(t))+M_{\kappa^{\prime}}(u(t))\right),
\end{aligned}
$$

where we have used (4.2), (4.3) and (4.5) at the second inequality. The proof of Lemma 5.2 has been completed.

LEMMA 5.3. (i) Let $n=3$ and $\kappa \geq 5$. Suppose that, for a local smooth solution $u$ of (3.1) and (3.2), the supremum of $E_{\kappa}^{1 / 2}(u(t))$ on an interval $[0, T)$

$$
\varepsilon_{0}:=\sup _{0 \leq t<T} E_{\kappa}^{1 / 2}(u(t))
$$

is sufficiently small. Then

$$
M_{\kappa}(u(t)) \leq C E_{\kappa}^{1 / 2}(u(t)), \quad 0 \leq t<T
$$

holds.

(ii) Let $n \geq 4$ and let $\kappa$ be large so that

$$
\left[\frac{\kappa-1}{2}\right]+\left[\frac{n}{2}\right]+2 \leq \kappa-1-\left[\frac{n}{2}\right] .
$$


Set $\mu=\kappa-1-[n / 2]$. Suppose that, for a local smooth solution $u$ of (3.1) and (3.2), the supremum of $E_{\mu}^{1 / 2}(u(t))$ on an interval

$$
\varepsilon_{0}:=\sup _{0 \leq t<T} E_{\mu}^{1 / 2}(u(t))
$$

is sufficiently small. Then the following hold:

$$
\begin{array}{ll}
M_{\mu}(u(t)) \leq C E_{\mu}^{1 / 2}(u(t)), & 0 \leq t<T, \\
M_{\kappa}(u(t)) \leq C E_{\kappa}^{1 / 2}(u(t)), & 0 \leq t<T .
\end{array}
$$

Proof. Part (i) is an immediate consequence of (5.1) and (5.3). We may focus on the proof of Part (ii). Set

$$
\mu^{\prime}=\left[\frac{\mu-1}{2}\right]+\left[\frac{n}{2}\right]+2, \quad \kappa^{\prime}=\left[\frac{\kappa-1}{2}\right]+\left[\frac{n}{2}\right]+2 .
$$

Recalling (5.11), we see simply but crucially $\mu^{\prime} \leq \kappa^{\prime} \leq \mu \leq \kappa$. We first employ (5.1) and (5.3), setting $\kappa \equiv \mu$ in the notation of lemmas 5.1 and 5.2, to get

$$
\begin{aligned}
M_{\mu}(u(t)) \leq & C E_{\mu}^{1 / 2}(u(t))+C \sum_{|a| \leq \mu-2}\left\|(t+r) \square \Gamma^{a} u(t)\right\|_{L^{2}} \\
\leq & C E_{\mu}^{1 / 2}(u(t))+C E_{\mu^{\prime}}^{1 / 2}(u(t)) E_{\mu}^{1 / 2}(u(t)) \\
& +C M_{\mu^{\prime}}(u(t)) E_{\mu}^{1 / 2}(u(t))+C E_{\mu^{\prime}}^{1 / 2}(u(t)) M_{\mu}(u(t)) \\
\leq & C E_{\mu}^{1 / 2}(u(t))+C \varepsilon_{0} E_{\mu}^{1 / 2}(u(t))+C \varepsilon_{0} M_{\mu}(u(t)),
\end{aligned}
$$

which yields (5.13). Getting back to (5.1) and (5.3) and noting $\kappa^{\prime} \leq \mu$, we find

$$
\begin{aligned}
M_{\kappa}(u(t)) \leq & C E_{\kappa}^{1 / 2}(u(t))+C \sum_{|a| \leq \kappa-2}\left\|(t+r) \square \Gamma^{a} u(t)\right\|_{L^{2}} \\
\leq & C E_{\kappa}^{1 / 2}(u(t))+C E_{\kappa^{\prime}}^{1 / 2}(u(t)) E_{\kappa}^{1 / 2}(u(t)) \\
& +C M_{\kappa^{\prime}}(u(t)) E_{\kappa}^{1 / 2}(u(t))+C E_{\kappa^{\prime}}^{1 / 2}(u(t)) M_{\kappa}(u(t)) \\
\leq & C E_{\kappa}^{1 / 2}(u(t))+C \varepsilon_{0} E_{\kappa}^{1 / 2}(u(t))+C \varepsilon_{0} M_{\kappa}(u(t)),
\end{aligned}
$$

which leads to (5.14). The proof has been completed.

6. Energy estimates $n=3$. Carrying out the estimates of the nonlinear term carefully, we shall find in the case $n=3$ that the generalized energy norm in (2.4), which contains at most one scaling operator $S$, indeed works well for our energy integral argument of the almost global existence. For the proof of the global existence theorem in higher dimensions, we shall get a pair of coupled differential inequalities for a higher-order energy $E_{\kappa}(u(t))$ and a lower-order energy $E_{\mu}(u(t)), \mu=\kappa-[n / 2]-1$. Since the equation (3.1) is quasi-linear, we must actually consider modified energies (see (6.1)) which are equivalent to the original ones for small solutions. Allowing the higher-order (modified) energy to grow polynomially in time but bounding the lower-order (modified) energy uniformly in time, we shall accomplish 
our energy integral argument. This strategy has already been employed in previous papers, such as Sideris and Tu [25].

Taking account of the difference between the definitions of the generalized energies (see (2.4)), we shall carry out the energy integral argument for $n=3$ in this section, and put it off for $n \geq 4$ until the next section.

Three space dimensions. Suppose $\kappa \geq 6$. For the initial data $(\varphi, \psi)$ with components $\left(\varphi^{k}, \psi^{k}\right) \in C_{0}^{\infty}\left(\boldsymbol{R}^{3}\right) \times C_{0}^{\infty}\left(\boldsymbol{R}^{3}\right)(k=1, \ldots, m)$, let us assume $E_{\kappa}^{1 / 2}(u(0))<\varepsilon$ for a sufficiently small $\varepsilon$ such that $3 \varepsilon \leq \varepsilon_{0}$ (as for $\varepsilon_{0}$, see (5.9)). By the standard local existence theorem we know that a unique smooth solution exists locally in time. Suppose that $T_{0}$ is the largest time such that $E_{\kappa}^{1 / 2}(u(t))<3 \varepsilon$ for $0 \leq t<T_{0}$. It is shown that $E_{\kappa}^{1 / 2}(u(t))<3 \varepsilon$ for $0 \leq t \leq A \exp [B / \varepsilon]$ for suitable constants $A$ and $B$ independent of $\varepsilon$. Therefore the existence time of the local solution exceeds $A \exp [B / \varepsilon]$.

Suppose $0 \leq t<T_{0}$ in what follows. Introducing the modified energy

$$
\tilde{E}_{\kappa}(u(t))=E_{\kappa}(u(t))-\frac{1}{2} \sum_{\substack{|a|=\kappa-1 \\|a 7| \leq 1}} \sum_{k=1}^{m} G_{i j}^{k, \alpha \beta \gamma} \eta_{\gamma}^{\delta} \int_{R^{3}} \partial_{\alpha} u^{i} \partial_{\beta} \Gamma^{a} u^{j} \partial_{\delta} \Gamma^{a} u^{k} d x
$$

$\left(\eta_{\gamma}^{\delta}=\operatorname{diag}(1,-1,-1,-1)\right)$ and following Sideris and Tu [25] on pages 484-485, we get

$$
\begin{aligned}
\tilde{E}_{\kappa}^{\prime}(u(t)) \leq & C \sum_{i, j, k} \sum_{\substack{|a| \leq \kappa-1 \\
|a 7| \leq 1}} \sum_{\substack{|b|+|c| \leq|a| c \neq c \neq a \\
b 7+c 7 \leq 1}}\left\|\partial \Gamma^{b} u^{i} \partial^{2} \Gamma^{c} u^{j}\right\|_{L^{2}}\left\|\partial \Gamma^{a} u^{k}\right\|_{L^{2}} \\
& +C \sum_{i, j, k} \sum_{\substack{|a| \leq \kappa-1 \\
|a 7| \leq 1}} \sum_{\substack{b|+| c|\leq| a \mid \\
b_{7}+c 7 \leq 1}}\left\|\partial \Gamma^{b} u^{i} \partial \Gamma^{c} u^{j}\right\|_{L^{2}}\left\|\partial \Gamma^{a} u^{k}\right\|_{L^{2}} .
\end{aligned}
$$

Since it follows easily from the Sobolev embedding that

$$
\frac{1}{2} E_{\kappa}(u(t)) \leq \tilde{E}_{\kappa}(u(t)) \leq 2 E_{\kappa}(u(t)), \quad 0 \leq t<T_{0}
$$

for the small solution $u$ under consideration, we may replace the norm $E_{\kappa}^{1 / 2}(u(t))$ with $\tilde{E}_{\kappa}^{1 / 2}(u(t))$ in the estimates below.

Set $q=[\kappa / 2]$ and $c_{0}=\min \left\{c_{j} / 2: j=1, \ldots, m\right\}$ as before. We start with the estimate of the first term on the right-hand side of (6.2), which is the contribution from the quasi-linear part.

Quasi-linear part. We separate two cases: $|b| \leq q$ or $|c| \leq q-1$.

Case $|b| \leq q . \quad$ If $\Gamma^{b}$ contains the operator $S$, then we have by (4.3)

$$
\begin{aligned}
\| \partial \Gamma^{b} & u^{i} \partial^{2} \Gamma^{c} u^{j} \|_{L^{2}} \\
& \leq C\langle t\rangle^{-1}\left(\left\|\partial \Gamma^{b} u^{i}\left\langle c_{j} t-r\right\rangle \partial^{2} \bar{\Gamma}^{c} u^{j}\right\|_{L^{2}\left(r<c_{0} t\right)}+\left\|\langle r\rangle \partial \Gamma^{b} u^{i} \partial^{2} \bar{\Gamma}^{c} u^{j}\right\|_{L^{2}\left(r>c_{0} t\right)}\right) \\
& \leq C\langle t\rangle^{-1}\left(\left\|\partial \Gamma^{b} u^{i}\right\|_{L^{\infty}}\left\|\left\langle c_{j} t-r\right\rangle \partial^{2} \bar{\Gamma}^{c} u^{j}\right\|_{L^{2}}+\left\|\langle r\rangle \partial \Gamma^{b} u^{i}\right\|_{L^{\infty}}\left\|\partial^{2} \bar{\Gamma}^{c} u^{j}\right\|_{L^{2}}\right) \\
& \leq C\langle t\rangle^{-1}\left(E_{|b|+3}^{1 / 2}(u(t)) M_{|c|+2}(u(t))+E_{|b|+3}^{1 / 2}(u(t)) E_{|c|+2}^{1 / 2}(u(t))\right) \\
& \leq C\langle t\rangle^{-1} E_{q+3}^{1 / 2}(u(t))\left(E_{\kappa}^{1 / 2}(u(t))+M_{\kappa}(u(t))\right) \leq C\langle t\rangle^{-1} E_{\kappa}(u(t)) .
\end{aligned}
$$


If $\Gamma^{b}$ does not contain $S$, then we obtain by (4.2) and (4.3)

$$
\begin{aligned}
& \left\|\partial \Gamma^{b} u^{i} \partial^{2} \Gamma^{c} u^{j}\right\|_{L^{2}} \\
& \leq C\langle t\rangle^{-1}\left(\left\|\left\langle c_{i} t-r\right\rangle \partial \bar{\Gamma}^{b} u^{i} \partial^{2} \Gamma^{c} u^{j}\right\|_{L^{2}\left(r<c_{0} t\right)}+\left\|\langle r\rangle \partial \bar{\Gamma}^{b} u^{i} \partial^{2} \Gamma^{c} u^{j}\right\|_{L^{2}\left(r>c_{0} t\right)}\right) \\
& \leq C\langle t\rangle^{-1}\left(\left\|\left\langle c_{i} t-r\right\rangle \partial \bar{\Gamma}^{b} u^{i}\right\|_{L^{\infty}}+\left\|\langle r\rangle \partial \bar{\Gamma}^{b} u^{i}\right\|_{\left.L^{\infty}\right)}\left\|\partial^{2} \Gamma^{c} u^{j}\right\|_{L^{2}}\right. \\
& \leq C\langle t\rangle^{-1}\left(E_{|b|+3}^{1 / 2}(u(t))+M_{|b|+3}(u(t))\right) E_{|c|+2}^{1 / 2}(u(t)) \leq C\langle t\rangle^{-1} E_{\kappa}(u(t)) \text {. }
\end{aligned}
$$

Case $|c| \leq q-1$. If $\Gamma^{b}$ contains $S$, then we easily have

$$
\begin{aligned}
\| \partial \Gamma^{b} & u^{i} \partial^{2} \Gamma^{c} u^{j} \|_{L^{2}} \\
& \leq C\langle t\rangle^{-1}\left(\left\|\partial \Gamma^{b} u^{i}\left\langle c_{j} t-r\right\rangle \partial^{2} \bar{\Gamma}^{c} u^{j}\right\|_{L^{2}\left(r<c_{0} t\right)}+\left\|\partial \Gamma^{b} u^{i}\langle r\rangle \partial^{2} \bar{\Gamma}^{c} u^{j}\right\|_{L^{2}\left(r>c_{0} t\right)}\right) \\
& \leq C\langle t\rangle^{-1}\left\|\partial \Gamma^{b} u^{i}\right\|_{L^{2}}\left(\left\|\left\langle c_{j} t-r\right\rangle \partial^{2} \bar{\Gamma}^{c} u^{j}\right\|_{L^{\infty}}+\left\|\langle r\rangle \partial^{2} \bar{\Gamma}^{c} u^{j}\right\|_{\left.L^{\infty}\right)}\right. \\
& \leq C\langle t\rangle^{-1} E_{|b|+1}^{1 / 2}(u(t))\left(E_{|c|+4}^{1 / 2}(u(t))+M_{|c|+4}(u(t))\right) \\
& \leq C\langle t\rangle^{-1} E_{\kappa}^{1 / 2}(u(t))\left(E_{q+3}^{1 / 2}(u(t))+M_{q+3}(u(t))\right) \leq C\langle t\rangle^{-1} E_{\kappa}(u(t)) .
\end{aligned}
$$

If $\Gamma^{b}$ does not contain $S$, then we see, noting $|b| \leq \kappa-2$ in this case,

$$
\begin{aligned}
\left\|\partial \Gamma^{b} u^{i} \partial^{2} \Gamma^{c} u^{j}\right\|_{L^{2}} & \leq C\langle t\rangle^{-1}\left(\left\|\frac{1}{r}\left\langle c_{i} t-r\right\rangle \partial \bar{\Gamma}^{b} u^{i} r \partial^{2} \Gamma^{c} u^{j}\right\|_{L^{2}\left(r<c_{0} t\right)}+\left\|\partial \bar{\Gamma}^{b} u^{i}\langle r\rangle \partial^{2} \Gamma^{c} u^{j}\right\|_{L^{2}\left(r>c_{0} t\right)}\right) \\
(6.7) \quad & \leq C\langle t\rangle^{-1}\left(\left\|\frac{1}{r}\left\langle c_{i} t-r\right\rangle \partial \bar{\Gamma}^{b} u^{i}\right\|_{L^{2}}+\left\|\partial \bar{\Gamma}^{b} u^{i}\right\|_{L^{2}}\right)\left\|\langle r\rangle \partial^{2} \Gamma^{c} u^{j}\right\|_{L^{\infty}} \\
& \leq C\langle t\rangle^{-1}\left(E_{|b|+1}^{1 / 2}(u(t))+M_{|b|+2}(u(t))\right) E_{|c|+4}^{1 / 2}(u(t)) \\
& \leq C\langle t\rangle^{-1}\left(E_{\kappa-1}^{1 / 2}(u(t))+M_{\kappa}(u(t))\right) E_{q+3}^{1 / 2}(u(t)) \leq C\langle t\rangle^{-1} E_{\kappa}(u(t)),
\end{aligned}
$$

where we have used the Hardy inequality at the third inequality.

Semi-linear part. In estimating the second term on the right-hand side of (6.2), we may assume $|b| \leq q(q=[\kappa / 2])$ without loss of generality. If $\Gamma^{b}$ contains the operator $S$, then we have, noting $|c| \leq \kappa-2$ in this case,

$$
\begin{aligned}
\left\|\partial \Gamma^{b} u^{i} \partial \Gamma^{c} u^{j}\right\|_{L^{2}} & \leq C\langle t\rangle^{-1}\left(\left\|r \partial \Gamma^{b} u^{i} \frac{1}{r}\left\langle c_{j} t-r\right\rangle \partial \bar{\Gamma}^{c} u^{j}\right\|_{L^{2}\left(r<c_{0} t\right)}+\left\|\langle r\rangle \partial \Gamma^{b} u^{i} \partial \bar{\Gamma}^{c} u^{j}\right\|_{L^{2}\left(r>c_{0} t\right)}\right) \\
& \leq C\langle t\rangle^{-1}\left\|\langle r\rangle \partial \Gamma^{b} u^{i}\right\|_{L^{\infty}}\left(\left\|\frac{1}{r}\left\langle c_{j} t-r\right\rangle \partial \bar{\Gamma}^{c} u^{j}\right\|_{L^{2}}+\left\|\partial \bar{\Gamma}^{c} u^{j}\right\|_{L^{2}}\right) \\
& \leq C\langle t\rangle^{-1} E_{|b|+3}^{1 / 2}(u(t))\left(E_{|c|+1}^{1 / 2}(u(t))+M_{|c|+2}(u(t))\right) \\
& \leq C\langle t\rangle^{-1} E_{q+3}^{1 / 2}(u(t))\left(E_{\kappa-1}^{1 / 2}(u(t))+M_{\kappa}(u(t))\right) \leq C\langle t\rangle^{-1} E_{\kappa}(u(t)) .
\end{aligned}
$$


If $\Gamma^{b}$ does not contain $S$, then, using (4.2), we get

$$
\begin{aligned}
\| \partial \Gamma^{b} & u^{i} \partial \Gamma^{c} u^{j} \|_{L^{2}} \\
& \leq C\langle t\rangle^{-1}\left(\left\|\left\langle c_{i} t-r\right\rangle \partial \bar{\Gamma}^{b} u^{i} \partial \Gamma^{c} u^{j}\right\|_{L^{2}\left(r<c_{0} t\right)}+\left\|\langle r\rangle \partial \bar{\Gamma}^{b} u^{i} \partial \Gamma^{c} u^{j}\right\|_{L^{2}\left(r>c_{0} t\right)}\right) \\
& \leq C\langle t\rangle^{-1}\left(\left\|\left\langle c_{i} t-r\right\rangle \partial \bar{\Gamma}^{b} u^{i}\right\|_{L^{\infty}}+\left\|\langle r\rangle \partial \bar{\Gamma}^{b} u^{i}\right\|_{\left.L^{\infty}\right)\left\|\partial \Gamma^{c} u^{j}\right\|_{L^{2}}}\right. \\
& \leq C\langle t\rangle^{-1}\left(E_{|b|+3}^{1 / 2}(u(t))+M_{|b|+3}(u(t))\right) E_{|c|+1}^{1 / 2}(u(t)) \\
& \leq C\langle t\rangle^{-1} E_{q+3}^{1 / 2}(u(t)) E_{\kappa}^{1 / 2}(u(t)) \leq C\langle t\rangle^{-1} E_{\kappa}(u(t)) .
\end{aligned}
$$

Conclusion of the proof. Using the equivalence (6.3), we have from (6.2), (6.4) through (6.9)

$$
\tilde{E}_{\kappa}^{\prime}(u(t)) \leq C \varepsilon\langle t\rangle^{-1} \tilde{E}_{\kappa}(u(t)), \quad 0 \leq t<T_{0},
$$

which yields

$$
\frac{1}{2} E_{\kappa}(u(t)) \leq \tilde{E}_{\kappa}(u(t)) \leq \tilde{E}_{\kappa}(u(0))(1+t)^{C \varepsilon} \leq 2 E_{\kappa}(u(0))(1+t)^{C \varepsilon} .
$$

Therefore,

$$
E_{\kappa}^{1 / 2}(u(t)) \leq 2 E_{\kappa}^{1 / 2}(u(0))(1+t)^{B \varepsilon}<2 \varepsilon(1+t)^{B \varepsilon}
$$

for a suitable constant $B$. Choosing $t$ so that $2(1+t)^{B \varepsilon} \leq 3$, we see $E_{\kappa}^{1 / 2}(u(t))<3 \varepsilon$, which implies that there exist suitable constants $A$ and $B$, and the existence time of the local solution exceeds $A \exp [B / \varepsilon]$ for all small $\varepsilon>0$. We have completed the proof of the theorem for $n=3$.

7. Energy estimates $n \geq 4$. Choose an integer $\kappa$ large so that

$$
\left[\frac{\kappa}{2}\right]+\left[\frac{n}{2}\right]+2 \leq \kappa-\left[\frac{n}{2}\right]-1
$$

Set $\mu=\kappa-[n / 2]-1$. Let us assume that the initial data satisfy

$$
E_{\mu}^{1 / 2}(u(0)) \exp \left[C_{0} E_{\kappa}^{1 / 2}(u(0))\right]<\frac{3}{4} \varepsilon
$$

for a sufficiently small $\varepsilon$ such that $2 \varepsilon \leq \varepsilon_{0}$ (see (5.10) as for $\varepsilon_{0}$ ), where $C_{0}$ is a constant appearing in (7.18) below. Let us spell out the plan of our energy integral argument. Suppose that $T_{0}$ is the largest time such that the local solution satisfies $E_{\mu}^{1 / 2}(u(t))<2 \varepsilon$ for $0 \leq t<T_{0}$. It will be shown that $E_{\mu}^{1 / 2}(u(t))<2 \varepsilon$ for $0 \leq t \leq T_{0}$. Therefore we can continue the local solution to all time.

Suppose $0 \leq t<T_{0}$ in what follows. As in Sideris [24], and Sideris and Tu [25], we carry out the energy integral argument by deriving a pair of coupled differential inequalities for a higher-order energy $E_{\kappa}(u(t))$ and a lower-order energy $E_{\mu}(u(t))$. As we did in the previous section, it is of course necessary to introduce the modified energy

$$
\tilde{E}_{l}(u(t))=E_{l}(u(t))-\frac{1}{2} \sum_{|a|=l-1} \sum_{k=1}^{m} G_{i j}^{k, \alpha \beta \gamma} \eta_{\gamma}^{\delta} \int_{\boldsymbol{R}^{n}} \partial_{\alpha} u^{i} \partial_{\beta} \Gamma^{a} u^{j} \partial_{\delta} \Gamma^{a} u^{k} d x
$$


$\left(\eta_{\gamma}^{\delta}=\operatorname{diag}(1,-1, \ldots,-1)\right)$ for $l=1,2, \ldots, \kappa$. We note that it is easy to verify

$$
\frac{1}{2} E_{l}(u(t)) \leq \tilde{E}_{l}(u(t)) \leq 2 E_{l}(u(t)), \quad l=\mu, \kappa
$$

under the smallness of the lower-order energy $E_{\mu}^{1 / 2}(u(t))$. Allowing the higher-order energy $E_{\kappa}(u(t))$ to grow polynomially in time but bounding the lower-order one $E_{\mu}(u(t))$ uniformly in time, we complete the energy integral argument.

The starting point of the proof is the standard energy inequality

$$
\begin{aligned}
\tilde{E}_{l}^{\prime}(u(t)) \leq & C \sum_{i, j, k} \sum_{|a| \leq l-1} \sum_{\substack{|b|+|c| \leq|a| \\
c \neq a}}\left\|\partial \Gamma^{b} u^{i} \partial^{2} \Gamma^{c} u^{j}\right\|_{L^{2}}\left\|\partial \Gamma^{a} u^{k}\right\|_{L^{2}} \\
& +C \sum_{i, j, k} \sum_{|a| \leq l-1} \sum_{|b|+|c| \leq|a|}\left\|\partial \Gamma^{b} u^{i} \partial \Gamma^{c} u^{j}\right\|_{L^{2}}\left\|\partial \Gamma^{a} u^{k}\right\|_{L^{2}} .
\end{aligned}
$$

Note that we may use Lemma 5.3 (ii) freely below, because (5.11) is automatically satisfied thanks to (7.1).

Higher-order energy. Choose $l=\kappa$ in (7.5) and set $q=[\kappa / 2]$. We have a simple but crucial inequality $q+[n / 2]+2 \leq \mu$ because of (7.1). Set $c_{0}=\min \left\{c_{j} / 2: j=1, \ldots, m\right\}$ as before. The right-hand side of (7.5) is estimated as in the proof of (5.6) through (5.8). Therefore our proof here is a little sketchy.

We start with the estimate of the second term on the right-hand side of (7.5). Assuming $|b| \leq q$ without loss of generality, we have

$$
\begin{aligned}
\| \partial \Gamma^{b} & u^{i} \partial \Gamma^{c} u^{j} \|_{L^{2}} \\
& \leq C\langle t\rangle^{-1}\left(\left\|\left\langle c_{i} t-r\right\rangle \partial \Gamma^{b} u^{i} \partial \Gamma^{c} u^{j}\right\|_{L^{2}\left(r<c_{0} t\right)}+\left\|\langle r\rangle \partial \Gamma^{b} u^{i} \partial \Gamma^{c} u^{j}\right\|_{L^{2}\left(r>c_{0} t\right)}\right) \\
& \leq C\langle t\rangle^{-1}\left(\left\|\left\langle c_{i} t-r\right\rangle \partial \Gamma^{b} u^{i}\right\|_{L^{\infty}}+\left\|\langle r\rangle \partial \Gamma^{b} u^{i}\right\|_{L^{\infty}}\right)\left\|\partial \Gamma^{c} u^{j}\right\|_{L^{2}} \\
& \leq C\langle t\rangle^{-1}\left(E_{q+[n / 2]+2}^{1 / 2}(u(t))+M_{q+[n / 2]+2}(u(t))\right) E_{\kappa}^{1 / 2}(u(t)) \\
& \leq C\langle t\rangle^{-1} E_{\mu}^{1 / 2}(u(t)) E_{\kappa}^{1 / 2}(u(t)) .
\end{aligned}
$$

For the estimate of the first term on the right-hand side of (7.5) we separate two cases: $|b| \leq q$ or $|c| \leq q-1$. In the former case we proceed as in (5.7) to obtain

$$
\begin{aligned}
\left\|\partial \Gamma^{b} u^{i} \partial^{2} \Gamma^{c} u^{j}\right\|_{L^{2}} \\
\quad \leq C\langle t\rangle^{-1}\left(\left\|\partial \Gamma^{b} u^{i}\left\langle c_{j} t-r\right\rangle \partial^{2} \Gamma^{c} u^{j}\right\|_{L^{2}\left(r<c_{0} t\right)}+\left\|\langle r\rangle \partial \Gamma^{b} u^{i} \partial^{2} \Gamma^{c} u^{j}\right\|_{L^{2}\left(r>c_{0} t\right)}\right) \\
\quad \leq C\langle t\rangle^{-1} E_{|b|+[n / 2]+2}^{1 / 2}(u(t))\left(M_{|c|+2}(u(t))+E_{|c|+2}^{1 / 2}(u(t))\right) \\
\quad \leq C\langle t\rangle^{-1} E_{\mu}^{1 / 2}(u(t)) E_{\kappa}^{1 / 2}(u(t)) .
\end{aligned}
$$


Otherwise, we have $|c| \leq q-1$ and

$$
\begin{aligned}
\| \partial \Gamma^{b} & u^{i} \partial^{2} \Gamma^{c} u^{j} \|_{L^{2}} \\
& \leq C\langle t\rangle^{-1}\left\|\partial \Gamma^{b} u^{i}\right\|_{L^{2}}\left(\left\|\left\langle c_{j} t-r\right\rangle \partial^{2} \Gamma^{c} u^{j}\right\|_{L^{\infty}}+\left\|\langle r\rangle \partial^{2} \Gamma^{c} u^{j}\right\|_{L^{\infty}}\right) \\
& \leq C\langle t\rangle^{-1} E_{|b|+1}^{1 / 2}(u(t))\left(E_{|c|+[n / 2]+3}^{1 / 2}(u(t))+M_{|c|+[n / 2]+3}(u(t))\right) \\
& \leq C\langle t\rangle^{-1} E_{\kappa}^{1 / 2}(u(t)) E_{\mu}^{1 / 2}(u(t))
\end{aligned}
$$

as in (5.8). Collecting (7.5) through (7.8), we finally have

$$
\tilde{E}_{\kappa}^{\prime}(u(t)) \leq C\langle t\rangle^{-1} E_{\mu}^{1 / 2}(u(t)) E_{\kappa}(u(t)), \quad 0 \leq t<T_{0} .
$$

Lower-order energy. Take $l=\mu$ in (7.5). Making use of the Hardy inequality effectively, we improve the decay estimates presented above, when $|a| \leq \mu$. Let $0<\eta<1 / 2$. Employing (4.2), (4.4), (4.5) and (4.12), we get for $|b|+|c| \leq \mu-1,|b| \leq[\mu / 2]$

$$
\begin{gathered}
\left\|\partial \Gamma^{b} u^{i} \partial \Gamma^{c} u^{j}\right\|_{L^{2}} \\
\leq C\langle t\rangle^{-1-\eta}\left(\left\|r\left\langle c_{i} t-r\right\rangle \partial \Gamma^{b} u^{i} \frac{1}{r}\left\langle c_{j} t-r\right\rangle \partial \Gamma^{c} u^{j}\right\|_{L^{2}\left(r<c_{0} t\right)}\right. \\
\left.\quad+\left\|\langle r\rangle^{1+\eta} \partial \Gamma^{b} u^{i} \partial \Gamma^{c} u^{j}\right\|_{L^{2}\left(r>c_{0} t\right)}\right) \\
\quad \times\left(\left\|\partial \Gamma^{c} u^{j}\right\|_{L^{2}}+\left\|\left\langle c_{j} t-r\right\rangle \partial^{2} \Gamma^{c} u^{j}\right\|_{L^{2}}\right) \\
\leq C\langle t\rangle^{-1-\eta}\left(\left\|r\left\langle c_{i} t-r\right\rangle \partial \Gamma^{b} u^{i}\right\|_{L^{\infty}}+\left\|\langle r\rangle^{1+\eta} \partial \Gamma^{b} u^{i}\right\|_{\left.L^{\infty}\right)}\right. \\
\quad \times C\langle t\rangle^{-1-\eta}\left(E_{|b|+[n / 2]+2}^{1 / 2}(u(t))+M_{|b|+[n / 2]+2}(u(t))\right)\left(E_{|c|+1}^{1 / 2}(u(t))+M_{|c|+2}(u(t))\right) \\
\leq C\langle t\rangle^{-1-\eta} E_{\mu}^{1 / 2}(u(t)) E_{\kappa}^{1 / 2}(u(t)) .
\end{gathered}
$$

For the estimate of the first term on the right-hand side of (7.5) we separate two cases: $|b| \leq$ $[\mu / 2]$ or $|c| \leq[\mu / 2]-1$. For the former case we proceed as

$$
\begin{gathered}
\left\|\partial \Gamma^{b} u^{i} \partial^{2} \Gamma^{c} u^{j}\right\|_{L^{2}} \\
\leq C\langle t\rangle^{-1-\eta}\left(\left\|\left\langle c_{i} t-r\right\rangle \partial \Gamma^{b} u^{i}\left\langle c_{j} t-r\right\rangle \partial^{2} \Gamma^{c} u^{j}\right\|_{L^{2}\left(r<c_{0} t\right)}\right. \\
\left.+\left\|\langle r\rangle^{1+\eta} \partial \Gamma^{b} u^{i} \partial^{2} \Gamma^{c} u^{j}\right\|_{L^{2}\left(r>c_{0} t\right)}\right) \\
\leq C\langle t\rangle^{-1-\eta}\left(\left\|\left\langle c_{i} t-r\right\rangle \partial \Gamma^{b} u^{i}\right\|_{L^{\infty}}\left\|\left\langle c_{j} t-r\right\rangle \partial^{2} \Gamma^{c} u^{j}\right\|_{L^{2}}\right. \\
\left.+\left\|\langle r\rangle^{1+\eta} \partial \Gamma^{b} u^{i}\right\|_{L^{\infty}}\left\|\partial^{2} \Gamma^{c} u^{j}\right\|_{L^{2}}\right) \\
\leq C\langle t\rangle^{-1-\eta}\left(E_{|b|+[n / 2]+2}^{1 / 2}(u(t))+M_{|b|+[n / 2]+2}(u(t))\right) \\
\times\left(E_{|c|+2}^{1 / 2}(u(t))+M_{|c|+2}(u(t))\right) \\
\leq C\langle t\rangle^{-1-\eta} E_{\mu}^{1 / 2}(u(t)) E_{\kappa}^{1 / 2}(u(t)) .
\end{gathered}
$$


Otherwise, we have $|c| \leq[\mu / 2]-1$ and

$$
\begin{gathered}
\left\|\partial \Gamma^{b} u^{i} \partial^{2} \Gamma^{c} u^{j}\right\|_{L^{2}} \\
\leq C\langle t\rangle^{-1-\eta}\left(\left\|\frac{1}{r}\left\langle c_{i} t-r\right\rangle \partial \Gamma^{b} u^{i} r\left\langle c_{j} t-r\right\rangle \partial^{2} \Gamma^{c} u^{j}\right\|_{L^{2}\left(r<c_{0} t\right)}\right. \\
\left.+\left\|\partial \Gamma^{b} u^{i}\langle r\rangle^{1+\eta} \partial^{2} \Gamma^{c} u^{j}\right\|_{L^{2}\left(r>c_{0} t\right)}\right) \\
\leq C\langle t\rangle^{-1-\eta}\left(\left\|\frac{1}{r}\left\langle c_{i} t-r\right\rangle \partial \Gamma^{b} u^{i}\right\|_{L^{2}}\left\|r\left\langle c_{j} t-r\right\rangle \partial^{2} \Gamma^{c} u^{j}\right\|_{L^{\infty}}\right. \\
\left.+\left\|\partial \Gamma^{b} u^{i}\right\|_{L^{2}}\left\|\langle r\rangle^{1+\eta} \partial^{2} \Gamma^{c} u^{j}\right\|_{L^{\infty}}\right) \\
\leq C\langle t\rangle^{-1-\eta}\left(E_{|b|+1}^{1 / 2}(u(t))+M_{|b|+2}(u(t))\right) \\
\times\left(E_{|c|+[n / 2]+3}^{1 / 2}(u(t))+M_{|c|+[n / 2]+3}(u(t))\right) \\
\leq C\langle t\rangle^{-1-\eta} E_{\kappa}^{1 / 2}(u(t)) E_{\mu}^{1 / 2}(u(t)) .
\end{gathered}
$$

Gathering (7.5), (7.10) through (7.12) together, we have shown

$$
\tilde{E}_{\mu}^{\prime}(u(t)) \leq C\langle t\rangle^{-1-\eta} E_{\kappa}^{1 / 2}(u(t)) E_{\mu}(u(t)) .
$$

Conclusion of the proof. It follows from (7.4) and (7.9) that

$$
\tilde{E}_{\kappa}^{\prime}(u(t)) \leq C \varepsilon\langle t\rangle^{-1} \tilde{E}_{\kappa}(u(t)), \quad 0 \leq t<T_{0},
$$

which yields

$$
\frac{1}{2} E_{\kappa}(u(t)) \leq \tilde{E}_{\kappa}(u(t)) \leq \tilde{E}_{\kappa}(u(0))\langle t\rangle^{C \varepsilon} \leq 2 E_{\kappa}(u(0))\langle t\rangle^{C \varepsilon}, \quad 0 \leq t<T_{0} .
$$

We insert the inequality $E_{\kappa}^{1 / 2}(u(t)) \leq 2 E_{\kappa}^{1 / 2}(u(0))\langle t\rangle^{C \varepsilon}\left(0 \leq t<T_{0}\right)$ into (7.13), thereby obtaining

$$
\tilde{E}_{\mu}^{\prime}(u(t)) \leq C\langle t\rangle^{-1-\eta+C \varepsilon} E_{\kappa}^{1 / 2}(u(0)) \tilde{E}_{\mu}(u(t)), \quad 0 \leq t<T_{0} .
$$

If necessary, we choose $\varepsilon$ still smaller so that $C \varepsilon<\eta$ holds above. We finally find

$$
\begin{aligned}
\frac{1}{2} E_{\mu}(u(t)) & \leq \tilde{E}_{\mu}(u(t)) \leq \tilde{E}_{\mu}(u(0)) \exp \left[C E_{\kappa}^{1 / 2}(u(0))\right] \\
& \leq 2 E_{\mu}(u(0)) \exp \left[C E_{\kappa}^{1 / 2}(u(0))\right], \quad 0 \leq t<T_{0} .
\end{aligned}
$$

The last inequality, combined with (7.2), proves

$$
E_{\mu}^{1 / 2}(u(t)) \leq 2 E_{\mu}^{1 / 2}(u(0)) \exp \left[C_{0} E_{\kappa}^{1 / 2}(u(0))\right]<\frac{3}{2} \varepsilon, \quad 0 \leq t<T_{0} .
$$

We therefore conclude that $E_{\mu}^{1 / 2}(u(t))<2 \varepsilon$ on the closed interval [0, $\left.T_{0}\right]$. The proof has been completed.

\section{REFERENCES}

[ 1 ] R. AGEMI, Global existence of nonlinear elastic waves, Invent. Math. 142 (2000), 225-250. 
[2] R. Agemi and K. Yokoyama, The null condition and global existence of solutions to systems of wave equations with different speeds. Advances in Nonlinear Partial Differential Equations and Stochastics, Ser. Adv. Math. Appl. Sci., vol. 48, World Sci. Publishing, River Edge, NJ, 1998, pp 43-86.

[3] Y. M. Chen And T. T. LI, Global classical solutions for nonlinear evolution equations, Pitman Monogr. Surveys Pure Appl. Math. 45, Longman Scientific and Technical, Harlow, 1992.

[ 4 ] K. Hidano, Small data scattering and blow-up for a wave equation with a cubic convolution, Funkcial. Ekvac. 43 (2000), 559-588.

[5] K. Hidano, Scattering problem for the nonlinear wave equation in the finite energy and conformal charge space, J. Funct. Anal. 187 (2001), 274-307.

[ 6 ] K. HidAno, The global existence theorem for quasi-linear wave equations with multiple speeds, to appear in Hokkaido Math. J.

[ 7 ] L. Hörmander, Lectures on nonlinear hyperbolic differential equations, Math. Appl. 26, Springer, Paris, 1997.

[ 8 ] A. Hoshiga And H. Kubo, Global small amplitude solutions of nonlinear hyperbolic systems with a critical exponent under the null condition, SIAM J. Math. Anal. 31 (2000), 486-513.

[9] F. John, Blow-up for quasilinear wave equations in three space dimensions, Comm. Pure Appl. Math. 34 (1981), 29-51.

[10] F. John, Almost global existence of elastic waves of finite amplitude arising from small initial disturbances, Comm. Pure Appl. Math. 41 (1988), 615-666.

[11] F. John, Nonlinear Wave Equations, Formations of Singulalities, Univ. Lecture Ser. 2, Amer. Math. Soc., Providence, RI, 1990.

[12] F. John And S. Klainerman, Almost global existence to nonlinear wave equations in three space dimensions, Comm. Pure Appl. Math. 37 (1984), 443-455.

[13] M. Keel, F. Smith And C. D. Sogge, Almost global existence for some semilinear wave equations, J. Anal. Math. 87 (2002), 265-279.

[14] M. Keel, F. Smith And C. D. Sogge, Almost global existence for quasilinear wave equations in three space dimensions, J. Amer. Math. Soc. 17 (2004), 109-153.

[15] S. Klainerman, Uniform decay estimate and the Lorentz invariance of the classical wave equations, Comm. Pure Appl. Math. 38 (1985), 321-332.

[16] S. Klainerman, The null condition and global existence to nonlinear wave equations, Nonlinear Systems of Partial Differential Equations in Applied Mathematics, Part 1 (Santa Fe, N. M., 1984), 293-326, Lectures in Appl. Math. 23, Amer. Math. Soc., Providence, RI, 1996.

[17] S. Klainerman, Remarks on the global Sobolev inequalities in the Minkowski space $\boldsymbol{R}^{n+1}$, Comm. Pure Appl. Math. 40 (1987), 111-117.

[18] S. Klainerman and T. C. Sideris, On almost global existence for nonrelativistic wave equations in $3 D$, Comm. Pure Appl. Math. 49 (1996), 307-321.

[19] M. Kovalyov, Resonance-type behaviour in a system of nonlinear wave equations, J. Differential Equations 77 (1989), 73-83.

[20] J. L. Metcalfe, Global existence for semilinear wave equations exterior to nontrapping obstacles, Houston J. Math. 30 (2004), 259-281.

[21] K. NAKANISHI, Unique global existence and asymptotic behaviour of solutions for wave equations with noncoercive critical nonlinearity, Comm. Partial Differential Equations 24 (1999), 185-221.

[22] R. RACKE, Lectures on nonlinear evolution equations, Initial value problems, Aspects Math. E19, Vieweg \& Sohn, Braunschweig, 1992.

[23] T. C. SIDERIS, The null condition and global existence of nonlinear elastic waves, Invent. Math. 123 (1996), 323-342.

[24] T. C. SidERIS, Nonresonance and global existence of prestressed nonlinear elastic waves, Ann. of Math. (2) 151 (2000), 849-874. 
[25] T. C. Sideris AND S.- Y. TU, Global existence for systems of nonlinear wave equations in $3 D$ with multiple speeds, SIAM J. Math. Anal. 33 (2002), 477-488.

[26] C. D. SogGe, Lectures on nonlinear wave equations, Monogr. Anal. II, Int. Press, Boston, MA, 1995.

[27] C. D. SogGe, Global existence for nonlinear wave equations with multiple speeds, Harmonic Analysis at Mount Holyoke (South Hadley, MA, 2001), 353-366, Contemp. Math. 320. Amer. Math. Soc., Providence, RI, 2003.

[28] W. A. Strauss, Nonlinear wave equations, CBMS Reg. Conf. Ser. Math. 73., Amer. Math. Soc., Providence, RI, 1989.

[29] K. YокоYама, Global existence of classical solutions to systems of wave equations with critical nonlinearity in three space dimensions, J. Math. Soc. Japan 52 (2000), 609-632.

DEPARTMENT OF MATHEMATICS

FACULTY OF EDUCATION

MIE UNIVERSITY

1515 KAMIHAMA, TSU

MIE 514-8507

JAPAN

E-mail address: hidano@edu.mie-u.ac.jp 